\title{
Cluster Regime - The New Regime Of Flowing Of Gas-Liquid Mixture In Vertical Columns (Based On Experimental Data)
}

\author{
A. Yu. Ozerov \\ Institute of Volcanology and Seismology, Far East Division, Russian Academy of Science, \\ Petropavlovsk-Kamchatsky, Russia,ozerov@ozerov.ru
}

\begin{abstract}
For the revealing of the reasons of occurrence of discrete volcanic explosions of basalt magma the Complex Apparatus for Modeling Basaltic Eruptions (CAMBE) has been developed. It consists of two major systems - modeling and recording. The device is 18 meters high. During experiments gas-saturated model liquid acts from the saturator to the vertical transparent hose in which arising two-phase structures and its kinetics are studied. The experiments resulted in detecting and describing a new, never before known, mode of gas-liquid two-phase flow in a vertical column - defined here as cluster regime, which is characterized by regular alteration of dense gas bubble clusters separated from each other by the liquid not containing free gas phase. The mechanism of the cluster regime formation is conditioned by the processes of blocking of the hose working section by one big bubble or several smaller ones. It has been demonstrated that liquid, bubble, cluster and slug regime are regularly sequential and present polymorphic modifications of gas-saturated liquids migrating within vertically oriented conduits. Analysis of data on explosions at volcanoes, given the obtained experimental data on the mechanism of this process, allows concluding that realization of cluster or slug regimes in volcanic crater produces basaltic explosions.
\end{abstract}

Keywords: gas-liquid two-phase flow, gas-saturated model liquid, vertical column, types of gas-hydrodynamic regimes, volcanic eruptions

\section{Introduction}

The subject of our studies includes a most frequently met type of volcanic activity Strombolian (Luchitsky, 1971; Macdonald, 1972; Rittmann, 1960). A Strombolian explosion is a powerful, abrupt and, as a rule, unexpected event. Explosions occur during summit, subsidiary and flank eruptions. An average in capacity basaltic explosion throws out to the surface about 30-50 tons of solid magmatic products, a strong one - 250-1000 tons.

Since there is no unambiguous universal explanation of the causes of Strombolian explosions we have conducted a cycle of experimental studies that allowed us to understand the mechanism of Strombolian activity. Laboratory experiments with twophase mixtures were started in 2002 and during 5 years we constructed 9 versions of gashydrodynamic experimental settings. As a result author has created Complex Apparatus for Modeling Basaltic Explosions - CAMBE.
The goal of the present study is to reveal the causes of discrete regime of eruption of basaltic magmas in the form of rhythmic explosions. Experimental studies with CAMBE included investigation of kinetics of gas-liquid twophase mixtures in vertical pipes (from the moment of nucleation of the first bubbles to formation of mature stable gas structures).

\section{Specification of Complex Apparatus for Modeling Basaltic Explosions and experimental research.}

While constructing the complex apparatus we did our best to consider the parameters of real feeding magma systems; the Klyuchevskoi volcano (Ozerov et al., 1997) which located on Kamchatka, Russia, has been accepted as a basis - a typical basaltic volcano. Besides we tried to avoid any possible structural and energy barriers, capable to affect the character of movement of the substance. The setting is constructed in the building of the Institute of 
Volcanology and Seismology, Far East Division, Russian Academy of Science. The overall height of CAMBE is 18 meters. The complex apparatus consists of two systems modeling and registrating (Fig. 1). It has been created for studying the volcanic processes, therefore along with technical terms in the description we will give volcanological notions that correspond to this or that section of CAMBE.

The modeling system includes a reservoir for preparation of gas-saturated model liquid (magma chamber), a transparent hose (feeding conduit), a plexiglass tank for reception of acting model liquid (crater area / vent) (Fig. 1). We shall give below the description of the parts of our apparatus from bottom to top:

"Chamber". When constructing this part of the apparatus we pursued the purpose to create a laboratory analogue of a magma chamber from which one-phase liquid with the dissolved gas feeds the conduit. The «Chamber» represents a thick-walled tight tank made of stainless steel with the height of 2,3 meters and capacity of 350 liters. The tank is designed for the pressure level up to 5 bars. With the help of a system of valves the system is filled with a liquid. For saturation of the liquid with gas, a punched tube with 50 apertures is welded in the bottom part of the tank. The pressure in the system is measured by a manometer which is located in the upper part of the tank. A water column located along the tank from the outside, allows one to monitor the volume of the liquid in the tank. In the upper part of the tank a connecting pipe is welded through which the inert gas is supplied to the tank during experiments; in our case it plays the role of squeezing out piston. Safety of the process of saturation of a liquid gas is ensured with an emergency safety valve. This part of the apparatus, acts as a matter of fact, as a saturator (the device that allows to dissolve gas in liquid).

In work we used a liquid and two kinds of gas. As a liquid we used water $\left(\mathrm{H}_{2} \mathrm{O}\right)$. As a dissolved gas we used carbonic gas $\left(\mathrm{CO}_{2}\right)$. It dissolves well in water (in 1 liter of $\mathrm{H}_{2} \mathrm{O}$ at temperature of the experiment of $20^{\circ} \mathrm{C}$ and

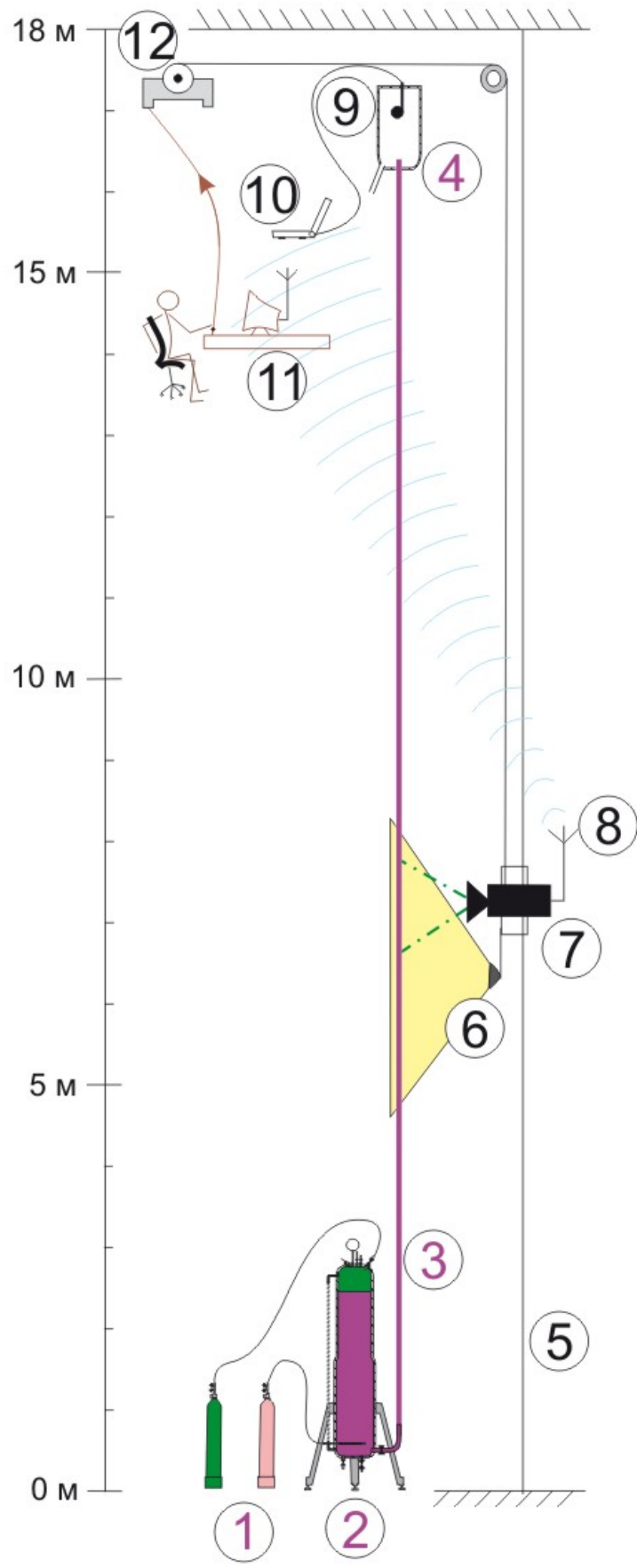

Fig. 1. A complex apparatus for modeling basaltic eruptions CAMBE.

a. Scheme of modeling (1-4) and recording (5-12) systems of CAMBE. 1 - high-pressure gas cylinders with $\mathrm{CO}_{2}$ and $\mathrm{N}_{2} ; 2$ - reservoir for preparation of a model liquid; 3 - transparent hose; 4 - an aquarium for reception of the liquid; 5 - a cable directing movement of a platform of dynamic video tracking; 6 light source; 7 - a video camera; 8 transmitter and the telemetering antenna; 9 - microphone; $10-\mathrm{a}$ computer; 11 - receiver of a video signal and a monitor; 12 - electric motor. In the upper part of the figure one can see the operator whose functions include adjustment of speed of movement of the block of video tracking.

pressure 1 bar, $828 \mathrm{ml}$ of $\mathrm{CO}_{2}$ is dissolved); besides, this gas easily transforms/exsolubles into a free phase (Namiot, 1991). Nitrogen $\left(\mathrm{N}_{2}\right)$ 
was used as a piston which squeezes out the model liquid from the tank. This gas at temperatures of the experiment is practically insoluble in water: in 1 liter of $\mathrm{H}_{2} \mathrm{O}$ at $\mathrm{T}=20^{\circ} \mathrm{C}$ only $18 \mathrm{ml}$ of $\mathrm{N}_{2}$ is dissolved (Namiot, 1991). Both gas cylinders supplying $\mathrm{CO}_{2}$ and $\mathrm{N}_{2}$, have reducers, that allow to adjust the discharge of gas.

«The conduit /Feeding column». In this part of the apparatus, processes occurring in the conduit of the volcano are modeled. From the bottom part of a side wall of the tank a horizontal pipe made of stainless steel is brought out which smoothly bending, changes the axial direction to the vertical. The smooth bend of the pipe is called to reduce effects of turbulence in the flow. The pipe is supplied with a locking spherical tap, which allows adjusting the supply of the model liquid from the tank to the plastic hose. The upper part of the steel pipe is attached to a transparent plastic hose; its length (height) - 16, $600 \mathrm{~mm}$, internal diameter $-18 \mathrm{~mm}$. The ratio of the working section: internal diameter of the hose to the length (in our case - height) is approximately 1: 1,000 ; such ratio is close to real parameters of volcanic conduits. The special system of fastening allows holding the hose in vertical position. Along the column a measuring tape with the scale division value of $1 \mathrm{~mm}$ is stretched. The pipe, the spherical tap and the hose have identical internal diameters. This, together with a smooth bend of the pipe, allows keeping a laminar character of the flow in the model liquid when it moves from the tank to the plastic hose.

«Crater area/vent ». The crater of a volcano or volcanic bocca is the natural analogue of this part of our complex. The upper part of the plastic hose is inserted into a transparent rectangular aquarium made of plexiglass. Sealing of it prevents the liquid to spill out of the CAMBE's limits. The aquarium is supplied with tank unloading. The hose rises above the bottom of the aquarium to $200 \mathrm{~mm}$; the cut of the hose at the top is made at the right angle to its axis.

The described part of our modeling system enables to trace the processes occurring during transition of the liquid from the feeding system to the open space.

Recording system. It includes a system of dynamic video tracking, an electronic altimeter and a speed meter, a block of video registration, a block of acoustic registration, a synchronizing device and a shutdown system (see Fig. 1).

The system of dynamic video tracking in real time monitors and registrates the processes occurring in the transparent vertical hose during movement of a model liquid in it. Along a hose fastened by a system of directing cables a cart with a video camera moves. Moving of the cart is provided with an electric motor. On the cart there is a powerful light source, allowing to discover even small heterogeneities in a moving flow. The image received by a video camera, in real time through a telemeter channel is transmitted to the monitor. The operator, who is monitoring the video image, using a control panel, has an opportunity to operatively change the speed of the cart in a range from $2 \mathrm{~cm} / \mathrm{s}$ up to $100 \mathrm{~cm} / \mathrm{s}$.

Video registration. The course of the experiment is being registered by video camera. The camera is located on the moving cart and records the picture of evolution of the model liquid rising in the transparent hose. For convenience of observation of the processes occurring in a hose, at the same time preserving the quality of the image, an optimum image capture angle was chosen, allowing to record a video series of moving bubbles in a $30-\mathrm{cm}$ interval of height.

The acoustic system allows registering the sound signal formed during destruction of bubbles, when they reach the surface of model liquid. For this purpose, above the top cut of the plastic hose a waterproofed microphone is attached, the signal from which in a digital form is transferred to the computer where it is recorded. It is a simulation of a geophysical station that records oscillations, arising near the vent of an erupting volcano.

The block of synchronization. This part of the complex apparatus is created for synchronization of video and acoustic information. It allows synchronizing a signal with the accuracy of a millisecond.

Shutdown system. The complex is supplied 
with the device of an emergency shutdown of the cart with a video camera in case of its getting out of the limits of the working zone.

It is necessary to specially emphasize, that in all experiments with CAMBE we deal with an open system.

As a result of preliminary experiments we have inferred that the full spectrum of gashydrodynamic regimes is achieved at saturation pressure of 1,6 bars. At this pressure we have conducted a series of experiments varying model liquid supply rate from 1 to $10 \mathrm{~cm} / \mathrm{s}$. The general structure of the flow and transition from one gas-hydrodynamic regime to another remained in these experiments similar.

During experiments while gas-saturated liquid moved in a vertical column we observed the reconstruction of the model liquid. We registered sequentially arising gashydrodynamic regimes - liquid, bubbly, cluster and slug. It is necessary to add that any of established gas-hydrodynamic regimes can be realized on the top cut depending on saturation pressure, which enables one to model different volcanic eruptions.

Special attention has been given to the earlier undescribed regime that unites a bubbly and slug regimes. This is a new, morphologically stable gas-hydrodynamic regime - a cluster regime.

\section{Discussion Of Results}

I. The results of experiments with CAMBE considerably supplement existing gashydrodynamic conceptions on which volcanological stipulations related to the dynamics of movement of magma melts are based. This can be explained by the fact that our experimental studies have some features which were not taken into account in earlier conducted works on hydrodynamic modeling of volcanic eruptions:

1. For the first time a model which attempted to reproduce geometrical parameters of the real volcano conduit has been realized. The length of real volcanic conduits is some orders of magnitude bigger that their diameter, therefore with CAMBE we have reproduced the ration of the inner diameter of the conduit to its length (height) $\sim 1: 1,000$.

2 . For the first time at physical modeling of eruptions conditions for ascent of gas-saturated liquid in a column have been created. This made it possible to observe the process of nucleation of bubbles, their subsequent growth, grouping into clusters and transformation into slugs.

3. The experiments were provided for with natural ascent of a model liquid; various structural barriers and fluctuations of supply rate of the gas-saturated liquid were excluded.

II. In the experiments one-phase model liquid turns into a two-phase system and in the course of evolution four regimes consistently and naturally (from below upwards) are realized: liquid, bubbly, cluster and slug (Fig. 2).

III. As a result of experimental studies, previously unknown necessary link has been established connecting bubbly and slug regimes. We suggest naming it a cluster regime (see Fig. 2, 13th and 14th meters). This is a new, morphologically steady gashydrodynamic regime. We will not be able to find the description of this regime in generalizing monographs by Wallis (1969), Kutateladze and Nakoryakov (1984), in Prandtl-Fihrer durch die Stromungslehre (2001) or in publications Abishev et al. (1981) and Sakharov and Mokhov (2004) on modeling works of oil wells on big size constructions.

We shall present below the basic characteristics of the cluster regime: 1 - the main component is a bubble cluster representing a volume of a liquid with high concentration of bubbles; from above and from below it is separated by a liquid containing no free gas phase; 2 - a series of bubble clusters following one after another at a certain distance, creates a cluster regime; 3 - it is always manifested between bubbly and slug regimes; 4 - cluster structures at certain column intervals have steady, repeating character; 5morphologically clusters of two types - open and blocked are distinguished; 6 - ascent rate of clusters is lower, than that of mature bubbly and slug regimes; 7 - life term of a cluster regime is comparable with that of bubbly and slug regimes; 8 - a cluster regime arises in a 
wide range of hydrodynamic conditions - in a bubble column (static and having no gas phase liquid), in the gas-saturated column (in the range of initial ascent rates from 1-10 cm/s); 9 - the mechanism of formation of clusters is related to the interaction of large gas structures with the walls of the channel, to the effects of self-locking and braking. a

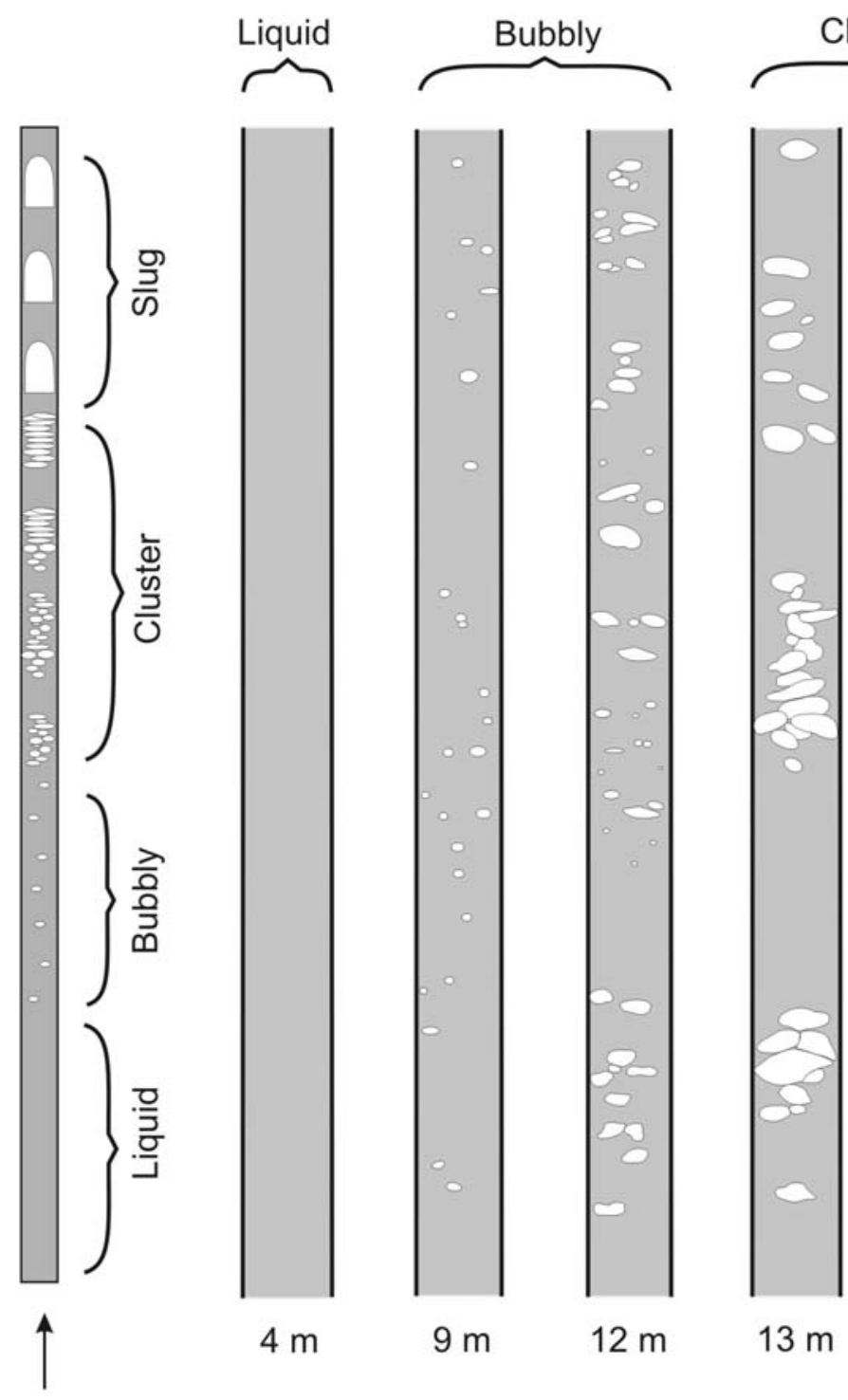

b
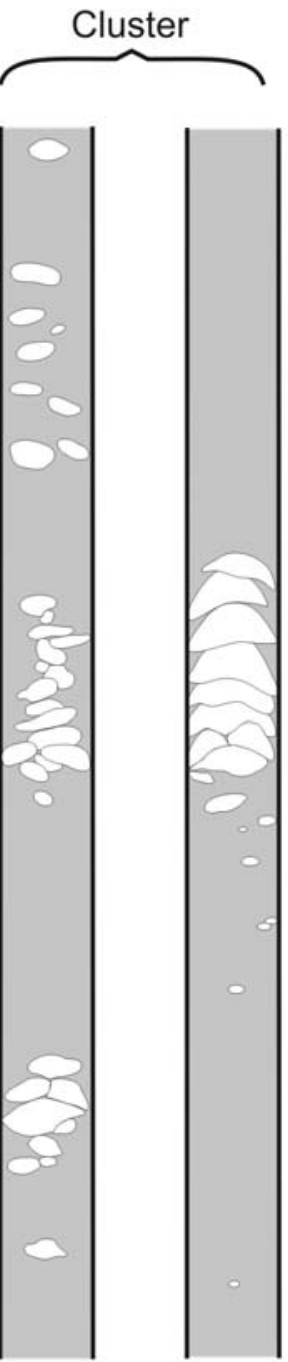

$14 \mathrm{~m}$
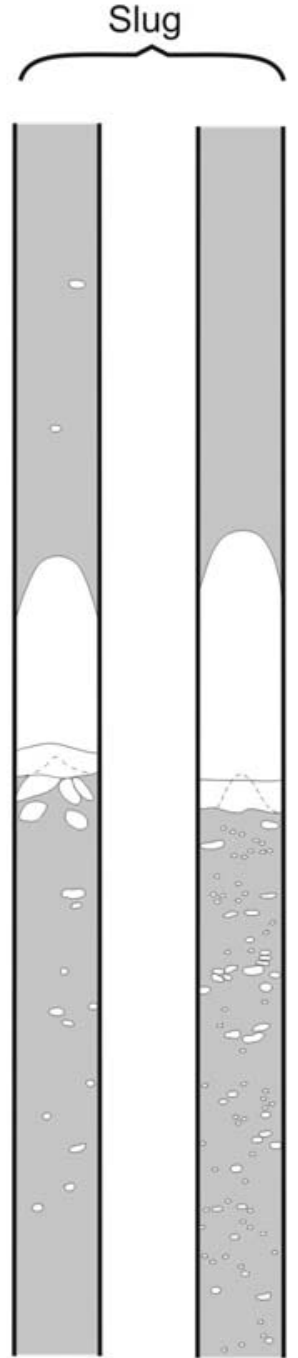

$30{ }^{\mathrm{cm}}$

$15 \mathrm{~m}$

Fig. 2. Fragments of the gas-hydrodynamic regimes originating during ascent of gas-saturated liquid in a vertical transparent hose. (a). Schematic view of location of gas-hydrodynamic regimes in the column;

(b). Snapshots of the gas-saturated model liquid flow regimes in the vertical channel. Every shot corresponds to $30 \mathrm{~cm}$ window of video recording. All shots of consequently evolving model liquid, were received during one travel of a video camera along the column, with the speed of a moving gas phase.

The author believes that a cluster regime is inherent not only in volcanic processes; it should be considered more widely, as an independent regime in the physics of gas-liquid mixtures, for example at natural objects (hydrothermal systems and mud volcanism), at modeling of boreholes (hydrothermal and oil) and in chemical industry.

IV. We shall describe now in more detail the mechanism of formation of cluster regime. The prototypes, "nucleuses" of future clusters are well traced already in the upper part of the column segment with the bubbly flow (see Fig. 2 , 12th meter). Non-uniform distribution of bubbles is observed here; clots, "swarms" or contractions consisting of individual bubbles are evident. In a segment of start of clustering these structures become more expressed. At 
some moment one large bubble or a contraction of smaller bubbles begin to interact with walls of the channel. The subsequent growth of the bubbles results in appreciable reduction of section of a return flow of the liquid - an annular clearance between gas structure and walls of the channel. An effect of self-locking takes place which results in slowered movement rate of a bubble /contraction "swarm" of bubbles. As a result, their ascent rate becomes lower, and it / they become an original "plug" for rising from below bubbles. The latter gather under the "plug", the distance between them decreases, and this results in the formation of a new gas structure out of a myriad of bubbles - a bubbly cluster (see Fig. 2, 13th and 14th meters). The bubbles in the column above the forming cluster, continue to move at original rate and rise upwards; thus a volume of liquid is formed containing no gas bubbles above the cluster. Same way liquid column segment without gas forms below the cluster. This is provided for by a new generating gas cluster forming at a lower level.

V. This process of transformation of clusters into slugs begins at the top part of a segment with mature cluster regime. It is characteristic only of the blocked clusters in which bubbles with their convex part directed upwards, are densely pressed to each other and with their boards stick to the internal walls of the channel. At such very dense dynamic packing the process of coalescence is inevitable and in the upper part of a cluster a large gas bubble is formed of smaller convex bubbles. Coalescence of bubbles begins (slug formation), it occurs from top to bottom in a cluster and within a few moments the whole gas cluster turns into classic gas slug (see Fig. 2,15 th meter).

The slug mode is the steadiest - in the conditions of our experiment. It can exist as long as you want and it would not transform into any other regime but the parameters of this regime during ascent also undergo certain changes. Hydrostatic pressure drop initiates diffusion of gas in the model liquid into slugs which results in their subsequent growing up. Their longitudinal sizes increase, their ascent rate grows. This leads to the formation of a relaxed zone in the rear part of a slug - in the bottom part of a slug a concave meniscus is clearly seen. Turbulence behind a slug amplifies. This gives start to a new stage of nucleation - the number of small bubbles behind the slug considerably increases (see Fig. 2,16 -th meter). Therefore, in a slug regime two sub-regimes are distinctly discriminated: initial - a pure slug when in the rear part of it practically no gas bubbles are present, and a mature slug in which every slug is accompanied by a train of fine bubbles.

VI. We shall describe now the ascent rate parameters of a two-phase flow whose evolution results in formation of clusters and slugs. As a result of our experimental studies it has been established, that structurization of a gas phase to gas clusters and slugs can occur in a wide range of ascent rates (initial rate of a liquid from 1 to $10 \mathrm{~cm} / \mathrm{s}$ ). This testifies to the fact that identified dynamic gas structures are integral in vertical gas-liquid systems and are realized in a wide speed range. This allows us to infer, that in real basaltic feeding conduits a gas phase undergoes similar structural evolution.

VII. Now we shall consider the display of each described hydrodynamic regime on the surface - on the top cut of the pipe because in this very part of CAMBE the processes occurring in the vent zone of basalt volcanoes are modeled. Depending on the quantity of the dissolved gas in a model liquid, every of the described regimes can be realized on the surface.

Liquid regime. Free phase gas is absent, uniform outpouring of a model liquid from the top cut of the pipe.

Bubbly regime results from uniform flow of bubbles, that burst on the surface.

Cluster regime is characterized by bursts of the model liquid as the bubble clusters reach the surface; bursts alternate with calm outpouring of a model liquid.

Slug regime - is characterized by abrupt bursts resulting from the break of a liquid layer above the gas slug reaching the surface; the bursts alternate with quiet outpouring or rise of liquid in the column.

Origination of gas cavities in magma in the 
form of clusters or slugs leads to significant redistribution of potential energy along magma column. The analysis of eruptions at different volcanoes shows that the strongest explosions take place with the viscosity of magma melts being within $10^{3}-10^{5} \mathrm{~Pa} \cdot \mathrm{s}$. The author assumes that at such viscosities the partitions in the cluster or in the layer of magma substance above the slug still preserve their elastic properties, but at the same time during instant liberation of potential energy of compressed gas at the exit of the gas structure (a cluster or a slug) to the surface behave as a solid body which results in high intensity of gas impulse up to real explosions.

\section{Conclusions}

1. To study the character of movement of magma melt in a feeding system of a volcano, the complex apparatus for modeling basaltic eruptions (CAMBE) has been constructed;

2 . The analysis of diversity of flow regimes of one and the same model liquid has shown that the four regimes of the flow - liquid, bubble, cluster and slug - result from natural evolution of gas saturated flow and that each of the regimes has its own specific features;

3. During experiments we have identified and described previously unknown regime of a two-phase mixture flow in a vertical column a cluster regime, characterized by natural alternation of dense concentrations of gas bubbles (clusters), separated from each other by a liquid containing no free gas phase. The mechanism of formation of clusters is accounted for by interaction of large gas structures with the walls of the channel as a result of which the effect of braking and selflocking arises and a new, slowly moving structure originates - gas plug - cluster;

4. A complex of studies accomplished has allowed us to offer a new model of gashydrodynamic evolutionary movement of a magma melt in the conduit of a basaltic volcano. Realization on the surface of this or that regime results in a variety of explosive events in a volcanic crater.

\section{Acknowledgements}

The author expresses sincere gratitude to Valerii A. Droznin for a long-term support of research. A big assistance to works was provided by the directorate of the Institute of Volcanology and Seismology, Far East Division, Russian Academy of Science: Academician Evgenii I. Gordeev, Academician Sergei A. Fedotov, Jaroslav D. Muraviev, and also my thanks to Alexei V. Butkach, Maxim G. Gavrilenko, Olga I. Dyachkova, Sergei V. Kasyanov, Anatolii P. Khrenov, Aleksandr S. Konov, Nina A. Ozerova, Vladimir S. Shulga, Ilia V. Chaplygin, Joels Richard Keith.

The preparation of the manuscript was supported by Academician Vitalii V. Adushkin, Academician Vyacheslav I. Kovalenko, Prof. Julii D. Chashechkin, Prof. Nikolaii N. Sysoev, Prof. John Eichelberger and Prof. Aleksandr A. Gusev.

The research was supported by grant of RFBR № 09-05-00841-a.

\section{References}

Abishev, S.K., $\quad$ Bulgakov, R.R., $\quad$ Sakharov, V.A., 1981. Experimental installation for research of movement of gasliquid mixtures in vertical pipes for ascent of high viscosity oils // Works MINH and GP, issue 156. Moscow. 98-104. (in Russian).

Kutateladze, S.S., Nakoryakov, V.E., 1984. Heat and mass exchange and waves in gas-liquid systems. Novosibirsk. Nauka. 302 p. (in Russian).

Luchitsky, I.V., 1971. The basics of palaeovolcanology. V 1. Modern volcanoes. Moscow. Nauka. 480 p. (in Russian).

Macdonald, G.A., 1972. Volcanoes. Prentice-Hall, Inc., Englewood Cliffs, New Jersey, $510 \mathrm{p}$.

Namiot, A.Yu., 1991. Solubility of gases in water. Moscow. $177 \mathrm{p}$.

Ozerov, A.Yu., Ariskin, A.A., Kyle, P., Bogoyavlenskaya, G.E., Karpenko, S.F., 1997, Petrologic-geochemical model of genetic relationship of basaltic and andesitic magmatism of Klyuchevskoy and Bezymianny volcanoes, Kamchatka. Petrology. Vol. 5, No. 6, 550-569. Translated from Petrologiya. Vol. 5, No. 6, 1997, 614-635.

Prandtl - Führer durch die Strömungslehre 2001. 718 p.

Rittmann, A., 1960. Vulkane und ihre tatigkeit. Ferdinand Enke Verlag, Stuttgart. $336 \mathrm{p}$.

Sakharov, V.A., Mokhov, M.A., 2004. Hydrodynamics of gasliquid mixtures in vertical pipes and field lifts. Publishing house Oil and gas. Moscow. 392 p. (in Russian).

Wallis, Graham B., 1969. One-dimensional two-phase flow. McGraw-Hill Book Company. 408 p. 